\title{
Original
}

\section{Dose-ranging efficacy of sitagliptin, a dipeptidyl peptidase-4 inhibitor, in Japanese patients with type 2 diabetes mellitus}

\author{
Yasuhiko Iwamoto $^{1)}$, Tadaaki Taniguchi ${ }^{2)}$, Kenji Nonaka $^{2)}$, Taro Okamoto ${ }^{2)}$, Kotoba Okuyama ${ }^{2}$, \\ Juan Camilo Arjona Ferreira ${ }^{3)}$ and John Amatruda ${ }^{3)}$ \\ ${ }^{1)}$ Diabetes Center, Tokyo Women's Medical University, Tokyo, Japan \\ ${ }^{2)}$ Banyu Pharmaceutical Co., LTD., Tokyo, Japan \\ ${ }^{3)}$ Merck \& Co., Inc., Rahway, NJ, United States
}

\begin{abstract}
Sitagliptin is an oral, potent, highly selective, once-daily DPP-4 inhibitor indicated for the treatment of type 2 diabetes mellitus (T2DM). To assess the dose-ranging efficacy and safety/tolerability profile of once-daily sitagliptin 25 , 50, 100, and $200 \mathrm{mg}$ in Japanese patients with T2DM. In this randomized, double-blind, placebo-controlled study, 363 Japanese patients with inadequate glycemic control $\left(\mathrm{HbA}_{1 \mathrm{c}}=6.5-10 \% ; \mathrm{FPG} \leq 270 \mathrm{mg} / \mathrm{dL}\right)$ were randomized $(1: 1: 1: 1: 1)$ to placebo, sitagliptin $25,50,100$, or $200 \mathrm{mg}$ q.d. for 12 weeks. The primary endpoint was change from baseline in $\mathrm{HbA}_{1 \mathrm{c}}$ at Week 12. At Week 12, treatment with sitagliptin at all doses tested provided significant $(p<0.001)$ reductions in $\mathrm{HbA}_{1 \mathrm{c}}$ $(-0.69$ to $-1.04 \%)$ from baseline $(7.49$ to $7.65 \%)$ relative to placebo. Sitagliptin significantly $(p<0.001)$ reduced fasting plasma glucose (FPG; -15.9 to $-23.2 \mathrm{mg} / \mathrm{dL}$ ) and 2-hour postprandial glucose (2-hr PPG; -40.3 to $-65.0 \mathrm{mg} / \mathrm{dL}$ ) relative to placebo, in a dose-dependent manner. At doses $\geq 50 \mathrm{mg}$, differences in $\mathrm{HbA}_{1 \mathrm{c}}$, FPG, and 2-hr PPG between the sitagliptin groups were not statistically significant. Sitagliptin was generally well tolerated with a low and similar incidence of hypoglycemia and minimal weight gain relative to placebo. Treatment with sitagliptin for 12 weeks provided significant and clinically meaningful reductions in $\mathrm{HbA}_{1 \mathrm{c}}, \mathrm{FPG}$, and 2-hr PPG across the dose range studied and was generally well tolerated in Japanese patients with T2DM.
\end{abstract}

Key words: Glycemic control, DPP-4 inhibitor, MK-0431, Incretins

THE INCRETIN HORMONES glucagon-like peptide-1 (GLP-1) and glucose-dependent insulinotropic polypeptide (GIP) are released by the gastrointestinal tract in response to a meal $[1,2]$. The intact forms of these peptides play an important role in regulating glucose homeostasis by stimulating both insulin production and glucose-dependent insulin secretion from pancreatic $\beta$-cells. GLP-1 further regulates plasma glucose levels by inhibiting glucagon secretion in a glucose-dependent manner, reducing food intake, and delaying gastric emptying. The actions of GLP-1 and

Received Sep. 17, 2009; Accepted Jan. 25, 2010 as K09E-272 Released online in J-STAGE as advance publication Mar. 24, 2010

Correspondence to: Juan Camilo ARJONA FERREIRA, Merck Research Laboratories, RY34-A232, 126 E. Lincoln Avenue, Rahway, NJ 07065, USA. Email: Juan_Arjona@merck.com

These data were previously presented at and published as an abstract for the $43^{\text {rd }}$ European Association for the Study of Diabetes (EASD), Sept 17-21, 2007.

Conflict of Interest: This study was supported by Merck Research
GIP in vivo are short-lived due to their rapid degradation and inactivation by the enzyme dipeptidyl peptidase-4 (DPP-4) [3, 4].

Sitagliptin is an oral, potent, highly selective, oncedaily DPP-4 inhibitor indicated for the treatment of type 2 diabetes mellitus (T2DM) [5]. Sitagliptin increases plasma levels of intact GLP-1 and GIP by twoto three-fold in patients with T2DM and in healthy volunteers[6-9]. In previously published studies conducted in non-Japanese patients with T2DM, treatment with sitagliptin was shown to reduce glycosylated he-

Laboratories, Merck \& Co., Inc. and Banyu Pharmaceutical Co., LTD.

Financial Disclosures: Yasuhiko Iwamoto has received lecture fees and research grants from Banyu Pharmaceutical Co., LTD. Authors Tadaaki Taniguchi, Kenji Nonaka, Taro Okamoto, and Kotoba Okuyama are or were employees of Banyu Pharmaceutical Co., LTD. and may own stock or hold stock options in the company. Authors Juan Camilo Arjona Ferreira and John Amatruda are or were employees of Merck \& Co., Inc. and may own stock or hold stock options in the company. 
moglobin $\left(\mathrm{HbA}_{1 \mathrm{c}}\right)$ as well as fasting and postprandial glucose levels in a dose-dependent manner [10, 11]. Sitagliptin is administered once daily as a single 100 -mg tablet in non-Japanese patients with normal to mildly impaired renal function,[12] as this dosing regimen was considered optimally effective in large multinational clinical dose-range finding studies $[10,11$, $13,14]$. Because sitagliptin is primarily renally excreted, sitagliptin dose adjustments to achieve plasma concentrations comparable to those in patients with normal renal function are recommended for patients with T2DM who have moderate renal insufficiency or severe renal insufficiency, including those with endstage renal disease requiring dialysis $[12,15,16]$.

In a recently published study, treatment with sitagliptin $100 \mathrm{mg}$ q.d. for 12 weeks improved fasting and postprandial glycemic control and was generally well-tolerated in Japanese patients with T2DM [17]. We have previously reported that sitagliptin possesses similar pharmacokinetic and pharmacodynamic profiles in healthy Japanese and non-Japanese subjects [7, $8,17]$. The aim of the current study was to evaluate the glycemic efficacy, dose response, and safety/tolerability profile of sitagliptin $25,50,100$, and $200 \mathrm{mg}$ administered once daily for 12 weeks in a population of Japanese patients with T2DM.

\section{Patients and Methods}

\section{Patients}

Male and female Japanese patients aged 20 to 75 years with T2DM were eligible to participate if they had inadequate glycemic control and, at screening, were either taking an oral anti-hyperglycemic agent (OHA) or had not taken an OHA for at least 8 weeks. Patients with type 1 diabetes, history of diabetic ketoacidosis, serum C-peptide $\leq 0.7 \mathrm{ng} / \mathrm{dL}$ at screening, treatment with insulin, PPAR $\gamma$ agonist (pioglitazone) or any combination OHA therapy in the 8 weeks prior to screening, unstable cardiac disease, active liver or gallbladder disease, inadequately controlled hypertension (systolic or diastolic blood pressure $>160$ $\mathrm{mm} \mathrm{Hg}$ or $>100 \mathrm{~mm} \mathrm{Hg}$, respectively), elevated serum creatinine $(>1.3 \mathrm{mg} / \mathrm{dL}$ in men and $>1.2 \mathrm{mg} / \mathrm{dL}$ in women), and elevations $>2$-fold the upper limit of normal (ULN) in alanine aminotransferase (ALT) and/ or aspartate aminotransferase (AST) were excluded. All patients provided written informed consent prior to the conduct of study procedures. The protocol
(Banyu Pharmaceutical Co., LTD. Protocol Number A202/Merck \& Co., Inc. Protocol Number 044) was reviewed and approved by the appropriate committees and authorities at each study site and conducted in accordance with the Declaration of Helsinki. This study was performed between the dates of July 11, 2005 and March 8, 2006.

\section{Study Design}

This Phase II, randomized, double-blind, placebocontrolled, parallel-arm study (www.clinicaltrials.gov registry number: NCT00127192) was conducted at 97 sites in Japan. At screening/Visit 1, the $\mathrm{HbA}_{1 \mathrm{c}}$ inclusion criteria ranged from $\geq 6.5 \%$ to $<10 \%$ for patients not on an OHA (for at least 8 weeks) and $\geq 6 \%$ to $\leq 9 \%$ for patients on OHA monotherapy (within 8 weeks prior to Visit 1). The study included a screening diet/ exercise run-in period of up to 8 weeks, including a 2-week single-blind placebo run-in period. At Visit 2, patients who had been on a diet and exercise program for at least 6 weeks and who had not taken an OHA for at least 6 weeks were eligible to directly enter the placebo run-in period. All other patients underwent a 6-week diet and exercise program (and wash-off period for patients on OHA at screening) prior to entering the placebo run-in period. This design ensured that patients received $\geq 8$ weeks of diet and exercise therapy without OHA treatment before randomization.

Patients with an $\mathrm{HbA}_{1 \mathrm{c}} \geq 6.5$ and $<10 \%$ and a fasting plasma glucose (FPG) $\leq 270 \mathrm{mg} / \mathrm{dL}$ upon entering the placebo run-in period and with $\geq 75 \%$ compliance with the single-blind placebo were randomized (1:1:1:1:1) to double-blind treatment with placebo or with sitagliptin $25 \mathrm{mg}, 50 \mathrm{mg}, 100 \mathrm{mg}$, or $200 \mathrm{mg}$ administered once daily before breakfast for 12 weeks. Patients were allocated to treatment assignment using an allocation schedule created by a Japanese thirdparty vendor that packaged the clinical supplies and provided them to the clinical site. Numbered containers were used to implement allocation and each patient was assigned the next number in the sequence upon being enrolled. All study personnel, including investigators, study site personnel, patients, monitors, and central laboratory personnel, remained blinded to treatment allocation throughout the study; the code was revealed to the researchers only after case report forms were completed, the database was locked, and statistical analysis plans were finalized. During the double-blind active treatment phase, patients received 
diet and exercise therapy. A follow-up phone call was made 14 days after the final dose of study medication to query patients regarding potential adverse experiences (AEs).

\section{Endpoints}

After an overnight fast, blood was collected for the assessment of $\mathrm{HbA}_{1 \mathrm{c}}$, FPG, 1,5-anhydroglucitol (1,5-AG), and glycosylated albumin (GA) at baseline (i.e., Week 0 prior to the first dose of study medication) and at various time points throughout the study. Homeostasis model assessment- $\beta$ cell (HOMA- $\beta$ ) was calculated to estimate pancreatic $\beta$-cell function. All patients were administered a standard meal tolerance test (MTT) at baseline and at Week 12. Patients took study medication 30 minutes prior to the standard meal, which was ingested within 15 minutes and contained $500 \mathrm{kcal}$ : $60 \%$ from carbohydrate, $15 \%$ from protein, and $25 \%$ from fat. Plasma glucose, insulin, and C-peptide concentrations were measured at 0,30 , 60 , and 120 minutes from the start of the meal for determination of 2-hour PPG, area under the glucose concentration-time curve (AUC), insulin AUC, and $\mathrm{C}$-peptide AUC. The insulinogenic index was measured by calculating the ratio of the change in postprandial insulin/glucose from baseline to 30 minutes [17]. Plasma glucagon levels were measured at 0,30 , 60 , and 120 minutes during the MTT in a subset of patients enrolled at clinic sites that were equipped to adequately collect and process the samples.

Safety and tolerability were evaluated from AE reports, vital signs (blood pressure, pulse rate and body weight), 12-lead electrocardiograms (ECGs), and laboratory tests, including hematology, blood chemistry, and urinalysis. AEs were rated by the investigators for the intensity and the potential relationship (definitely not, probably not, possibly, probably, or definitely) to study drug. AEs of special interest included hypoglycemia and selected gastrointestinal-related AEs (i.e., nausea, vomiting, and diarrhea). Patients were counseled to record results of self-monitored blood glucose levels and symptoms of hypoglycemia (e.g., sweating, anxiety, palpitations, headache, blurred vision, clouding of consciousness in diaries) for proper assessment of hypoglycemic events during the study. Hypoglycemia was assessed by the study site investigators through reviewing patient self-reports of signs and symptoms of hypoglycemia. A fingerstick blood glucose determination concurrent with the episode was not required to assess an episode as hypoglycemia, although investigators could include the fingerstick glucose measurement, if it was available, in their assessment of the episode.

All efficacy and safety laboratory measurements were assayed at a central laboratory (Mitsubishi Kagaku Bio-Clinical Laboratories [Mitsubishi Chemical Medience Corporation], Tokyo) by technicians blinded to treatment allocation.

\section{Statistical Analyses}

Efficacy analyses were performed on the full analysis set (FAS) consisting of all randomized patients who received at least one dose of study drug and who had valid measurements both at baseline and at least one post-treatment measurement. The primary efficacy endpoint, the change from baseline in $\mathrm{HbA}_{1 \mathrm{c}}$ at Week 12, was analyzed using an analysis of covariance (ANCOVA) model with terms for treatment, prior OHA use, and baseline $\mathrm{HbA}_{1 \mathrm{c}}$ value as covariates. The between-group differences (relative to placebo) and associated $95 \%$ confidence intervals (CIs) for efficacy endpoints were assessed by testing the differences in the least squares (LS) mean changes from baseline at Week 12. Missing data were handled using the last observation carried forward method. An alpha level of $\leq 0.050$ (two sided) was considered statistically significant. A stepwise linear contrast test based on the ANCOVA model was used to examine the dose-response relationship for placebo and the sitagliptin 25-, 50-, 100-, and 200-mg groups. LS mean differences between groups (relative to placebo) and associated $95 \%$ CI for each of the subgroups were estimated based on the aforementioned ANCOVA model to evaluate the consistency of the $\mathrm{HbA}_{1 \mathrm{c}}$-lowering effect of sitagliptin relative to placebo across predefined subgroups including baseline $\mathrm{HbA}_{1 \mathrm{c}}$ stratum $(\leq 8.0 \%$ or $>8.0 \%$ ).

Safety analyses were performed on the all-patients-as-treated population (APaT) consisting of all patients who took at least one dose of study drug. Summary statistics for changes from baseline in safety continuous parameters were calculated. For overall AEs, drug-related AEs, hypoglycemia, and selected gastrointestinal-related AEs (i.e., nausea, vomiting, and diarrhea), the comparisons with placebo were conducted by Fisher's exact test, and between-group differences (relative to placebo) and associated 95\% CIs were reported. 


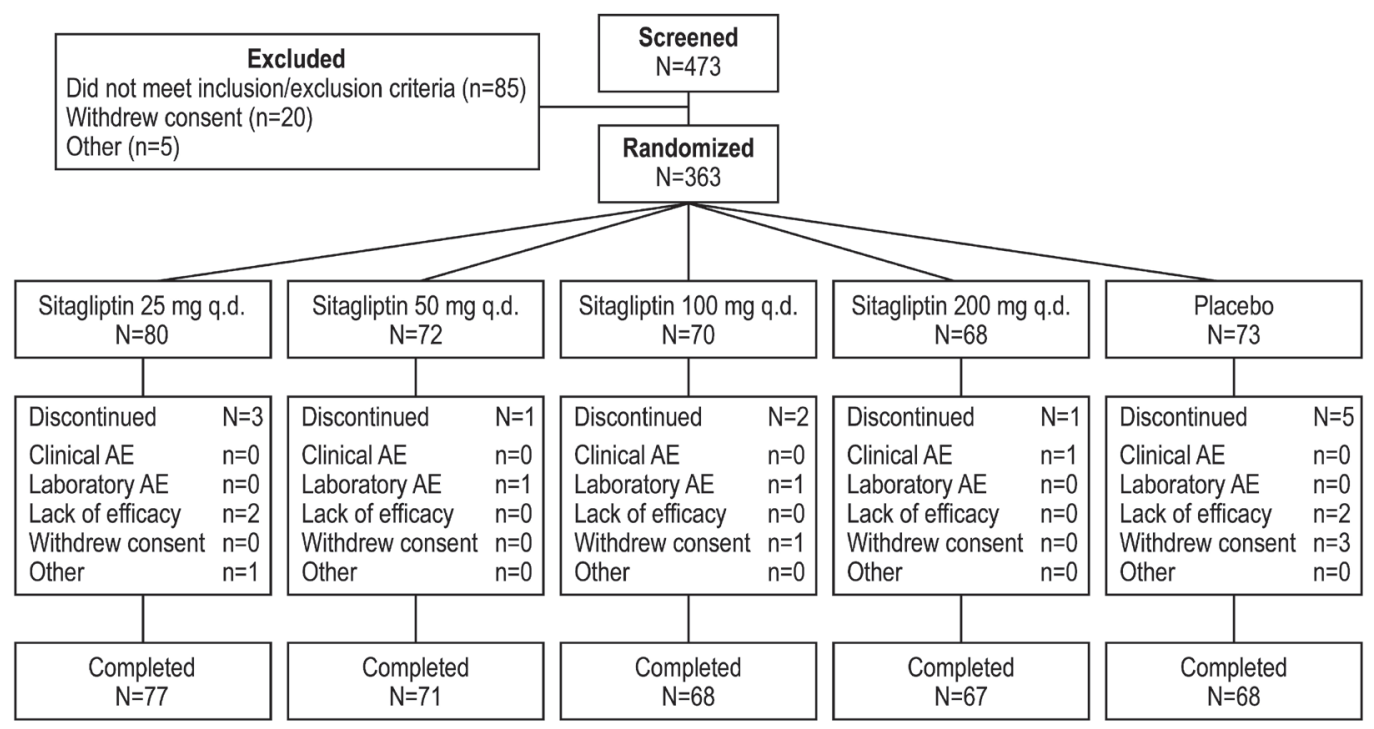

Fig. 1. Disposition of screened/randomized patients

Table 1. Summary of patient demographics and baseline characteristics

\begin{tabular}{|c|c|c|c|c|c|}
\hline \multirow[b]{2}{*}{ Parameter } & \multirow{2}{*}{$\begin{array}{l}\text { Placebo } \\
n=73\end{array}$} & \multicolumn{4}{|c|}{ Sitagliptin } \\
\hline & & $\begin{array}{c}25 \mathrm{mg} \text { q.d. } \\
\mathrm{n}=80\end{array}$ & $\begin{array}{c}50 \text { mg q.d. } \\
n=72\end{array}$ & $\begin{array}{c}100 \text { mg q.d. } \\
n=70\end{array}$ & $\begin{array}{c}200 \text { mg q.d. } \\
n=68\end{array}$ \\
\hline Age, yr & $60.2 \pm 8.0$ & $59.9 \pm 7.9$ & $60.2 \pm 9.4$ & $58.3 \pm 9.5$ & $60.6 \pm 7.7$ \\
\hline \multicolumn{6}{|l|}{ Gender, n (\%) } \\
\hline Women & $23(31.5)$ & $29(36.3)$ & $25(34.7)$ & 34 (48.6) & $28(41.2)$ \\
\hline Men & $50(68.5)$ & $51(63.8)$ & $47(65.3)$ & $36(51.4)$ & $40(58.8)$ \\
\hline BMI, $\mathrm{kg} / \mathrm{m}^{2}$ & $24.1 \pm 3.2$ & $25.0 \pm 3.6$ & $24.5 \pm 3.6$ & $24.2 \pm 2.8$ & $24.4 \pm 3.0$ \\
\hline Duration of diabetes, yr & $6.4 \pm 5.5$ & $4.7 \pm 4.3$ & $5.6 \pm 6.4$ & $5.4 \pm 5.4$ & $5.1 \pm 4.9$ \\
\hline \multicolumn{6}{|l|}{ Prior use of OHAs, n (\%) } \\
\hline Absence & $29(39.7)$ & $37(46.3)$ & $34(47.2)$ & $41(58.6)$ & $35(51.5)$ \\
\hline Presence & $44(60.3)$ & $43(53.8)$ & $38(52.8)$ & $29(41.4)$ & $33(48.5)$ \\
\hline $\mathrm{HbA}_{1 \mathrm{c}}, \%$ & $7.74 \pm 0.93$ & $7.49 \pm 0.82$ & $7.57 \pm 0.84$ & $7.56 \pm 0.80$ & $7.65 \pm 0.82$ \\
\hline $\mathrm{FPG}, \mathrm{mg} / \mathrm{dL}$ & $156.48 \pm 35.11$ & $145.71 \pm 37.58$ & $144.25 \pm 28.96$ & $142.60 \pm 31.77$ & $148.40 \pm 33.35$ \\
\hline
\end{tabular}

Data are expressed as mean \pm standard deviation or frequency unless otherwise noted.

$\mathrm{BMI}=$ body mass index; $\mathrm{FPG}=$ fasting plasma glucose $\mathrm{HbA}_{\mathrm{lc}}=$ glycosylated hemoglobin; $\mathrm{OHA}=$ oral antihyperglycemic agents; q.d. = once daily

\section{Results}

Of 473 patients screened for participation in this study, 110 were not randomized because they did not meet inclusion or met exclusion criteria $(\mathrm{n}=85)$, withdrew consent $(n=20)$, or other reason $(n=5)$. A total of 363 patients were randomized to receive doubleblind treatment with once-daily placebo $(\mathrm{n}=73)$, sitagliptin $25 \mathrm{mg}(\mathrm{n}=80), 50 \mathrm{mg}(\mathrm{n}=72), 100 \mathrm{mg}(\mathrm{n}=70)$, or $200 \mathrm{mg}(\mathrm{n}=68)$ for 12 weeks (Figure 1). Overall, $351(97 \%)$ patients completed the study, and 12 pa- tients discontinued before study completion. The discontinuation rate was somewhat higher in the placebo group relative to the sitagliptin groups, with withdrawal of consent and lack of efficacy being the most common reasons for discontinuation. All randomized patients contributed to the efficacy and safety analyses. Baseline demographics were generally well matched, and baseline glycemic characteristics were similar across the treatment groups (Table 1). Patients randomized in this study had a mean baseline $\mathrm{HbA}_{\mathrm{lc}}$ of $7.60 \%$ and FPG of $147.5 \mathrm{mg} / \mathrm{dL}$. The mean duration of diabetes was 5.4 years, and $51.5 \%$ of pa- 


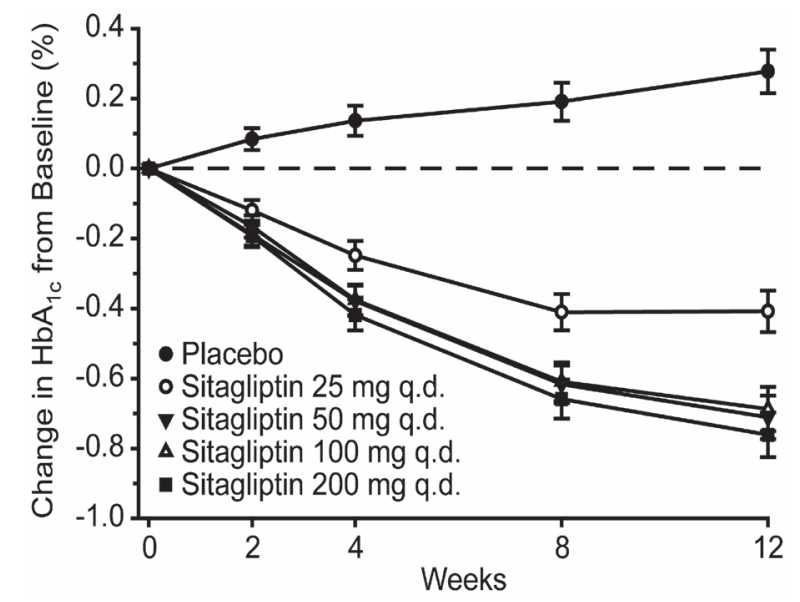

Fig. 2. Least squares mean change from baseline $( \pm \mathrm{SE})$ in $\mathrm{HbA}_{1 \mathrm{c}}$ over a 12-week treatment period.

tients had received prior OHA therapy within 8 weeks before initiation of the screening period.

\section{Efficacy}

At Week 12, all doses of sitagliptin produced significant reductions from baseline in $\mathrm{HbA}_{1 \mathrm{c}}(p<0.001)$ (Table 2). A significant dose-response relationship (as assessed by stepwise linear contrast test) in $\mathrm{HbA}_{1 \mathrm{c}}$ lowering was observed across all of the sitagliptin doses tested and placebo ( $p<0.001$ for all doses). Similar magnitude reductions from baseline in $\mathrm{HbA}_{1 \mathrm{c}}$ were seen across sitagliptin doses ranging from $50 \mathrm{mg}$ to $200 \mathrm{mg}$, with the 50-mg group, producing a placebo-subtracted change from baseline of $0.99 \%$ (95\% CI: -1.16, -0.82). No significant between-group differences in $\mathrm{HbA}_{1 \mathrm{c}}$ were seen for the 50-, 100-, and 200$\mathrm{mg}$ doses of sitagliptin. The $25-\mathrm{mg}$ dose produced significantly smaller reductions in $\mathrm{HbA}_{1 \mathrm{c}}$ compared with the other doses $(p<0.001 v s$. sitagliptin $25 \mathrm{mg}$ for all pair-wise comparisons). Over time, the placebo group showed a slow progressive increase from baseline in $\mathrm{HbA}_{1 \mathrm{c}}$ out to Week 12 (Figure 2). For the sitagliptin 25-mg group, maximum reductions from baseline in $\mathrm{HbA}_{1 \mathrm{c}}$ were reached by Week 8 and appeared stable out to Week 12. In contrast, the other sitagliptin groups showed a progressive reduction from baseline in $\mathrm{HbA}_{1 \mathrm{c}}$ out to Week 12 with no apparent plateau in the response. In the subgroup of patients with baseline $\mathrm{HbA}_{1 \mathrm{c}} \leq 8 \%$, sitagliptin doses of $50 \mathrm{mg}, 100 \mathrm{mg}$, and $200 \mathrm{mg}$ showed similar placebo-subtracted reductions from baseline in $\mathrm{HbA}_{1 \mathrm{c}}$ of $-0.76 \%$ (95\% CI: -0.93 , $-0.58),-0.80 \%$ (95\% CI: $-0.98,-0.63)$, and $-0.76 \%$
(95\% CI: -0.94, -0.59), respectively. In the subgroup of patients with baseline $\mathrm{HbA}_{1 \mathrm{c}}>8 \%$, treatment with sitagliptin $200 \mathrm{mg}$ produced the numerically largest placebo-subtracted reductions from baseline in $\mathrm{HbA}_{1 \mathrm{c}}$ of $-1.59 \%$ (95\% CI: $-1.97,-1.21)$, with substantial reductions of $-1.38 \%(95 \% \mathrm{CI}:-1.78,-0.99)$ in the 50 $\mathrm{mg}$ group and $-1.31 \%(95 \% \mathrm{CI}:-1.72,-0.89)$ in the 100-mg group.

Significant dose-dependent reductions from baseline in FPG were observed across the sitagliptin doses compared with placebo $(p<0.001$ for all doses) (Table 2; Figure 3). The reductions observed at doses of 100 and $200 \mathrm{mg}$ were numerically but not statistically superior to $50 \mathrm{mg}$. The only pairwise comparison that reached statistical significance was between the $25-\mathrm{mg}$ and $200-\mathrm{mg}$ groups $(p<0.050)$. Compared with placebo, sitagliptin also led to significant $(p<0.001$ for all doses of sitagliptin) dose-related increases from baseline in 1,5-AG and significant $(p<0.001$ for all doses of sitagliptin) dose-related reductions from baseline in GA (Table 2).

Relative to placebo, all doses of sitagliptin produced significant dose-related increases from baseline in HOMA- $\beta(p<0.050 v s$. placebo for all doses $)$ (Table 2). There were no significant differences in the HOMA- $\beta$ response among the 25-, 50-, 100-, and 200$\mathrm{mg}$ doses, except for a significant difference observed when the $25-\mathrm{mg}$ group was compared with the 100 $\operatorname{mg}$ group $(p=0.030)$.

With respect to the MTT parameters, treatment with sitagliptin across all doses tested led to significant reductions from baseline in 2-hr PPG and glucose $\mathrm{AUC}_{0-2}$ hr relative to placebo at Week $12(p<0.001 v s$. placebo for all doses), with the 200 -mg group providing the largest magnitude changes from baseline in both parameters (Table 2). No significant betweengroup differences in 2-hr PPG were seen between the sitagliptin 25- and 50-mg doses or among sitagliptin doses of 50, 100, and $200 \mathrm{mg}$. In contrast, the $25-\mathrm{mg}$ dose produced significantly smaller reductions in $2-\mathrm{hr}$ PPG compared with the $100-\mathrm{mg}(p=0.010)$ and 200$\mathrm{mg}$ doses $(p<0.001)$ (data not shown). No significant between-group differences in glucose prior to $\mathrm{AUC}_{0-2 \mathrm{hr}}$ were seen between sitagliptin doses of 50 and 100 $\mathrm{mg}$ or between sitagliptin doses of 100 and $200 \mathrm{mg}$; however, significantly smaller reductions in glucose $\mathrm{AUC}_{0-2 \mathrm{hr}}$ were observed with sitagliptin 25 $\mathrm{mg}$ compared with sitagliptin $50 \mathrm{mg}, 100 \mathrm{mg}$, and $200 \mathrm{mg}(p=0.026, p=0.004$ and $p<0.001$, respec- 
Table 2. Baseline, Week 12, and change from baseline in fasting and postprandial glycemic endpoints

\begin{tabular}{|c|c|c|c|c|c|}
\hline \multirow[b]{2}{*}{$\begin{array}{r}\text { Parameter } \\
\end{array}$} & \multirow[b]{2}{*}{ Placebo } & \multicolumn{4}{|c|}{ Sitagliptin } \\
\hline & & $25 \mathrm{mg}$ q.d. & $50 \mathrm{mg}$ q.d. & $100 \mathrm{mg}$ q.d. & $200 \mathrm{mg}$ q.d. \\
\hline \multicolumn{6}{|c|}{ Fasting Glycemic Endpoints } \\
\hline \multicolumn{6}{|c|}{$\mathrm{HbA}_{\mathrm{ic}}(\%)$} \\
\hline $\mathrm{n}$ & 73 & 80 & 72 & 70 & 68 \\
\hline Baseline $^{a}$ & $7.74(0.93)$ & $7.49(0.82)$ & $7.57(0.84)$ & $7.56(0.80)$ & $7.65(0.82)$ \\
\hline Week $12^{\mathrm{a}}$ & $8.04(1.24)$ & $7.11(0.94)$ & $6.87(0.82)$ & $6.85(0.90)$ & $6.88(0.80)$ \\
\hline Change from baseline ${ }^{\mathrm{b}}$ & $0.28(0.16,0.40)^{* *}$ & $-0.41(-0.52,-0.29)^{* *}$ & $-0.71(-0.83,-0.59)^{* *}$ & $-0.69(-0.81,-0.56)^{* *}$ & $-0.76(-0.89,-0.64)^{* *}$ \\
\hline Change from placebo ${ }^{c}$ & -- & $-0.69(-0.85,-0.52)^{\dagger \dagger}$ & $-0.99(-1.16,-0.82)^{\dagger \dagger}$ & $-0.96(-1.14,-0.79)^{\dagger \dagger}$ & $-1.04(-1.21,-0.86)^{\dagger \dagger}$ \\
\hline \multicolumn{6}{|c|}{ Fasting Plasma Glucose (mg/dL) } \\
\hline $\mathrm{n}$ & 73 & 80 & 72 & 70 & 68 \\
\hline Baseline $^{a}$ & $156.5(35.1)$ & $145.7(37.6)$ & $144.3(29.0)$ & $142.6(31.8)$ & $148.4(33.3)$ \\
\hline Week $12^{\mathrm{a}}$ & $159.5(33.2)$ & $136.9(27.0)$ & $134.3(30.0)$ & $129.7(26.9)$ & $131.1(25.1)$ \\
\hline Change from baseline ${ }^{b}$ & $6.3(1.7,10.9)^{*}$ & $-9.6(-14.0,-5.3)^{* *}$ & $-11.4(-16.0,-6.8)^{* *}$ & $-14.6(-19.2,-9.9)^{* *}$ & $-16.9(-21.6,-12.1)^{* *}$ \\
\hline Change from placebo ${ }^{c}$ & -- & $-15.9(-22.3,-9.6)^{\dagger \dagger}$ & $-17.7(-24.2,-11.2)^{\dagger \dagger}$ & $-20.8(-27.4,-14.3)^{\dagger \dagger}$ & $-23.2(-29.8,-16.6)^{\dagger \dagger}$ \\
\hline \multicolumn{6}{|c|}{ 1.5-Anhydroglucitol $(\mu \mathrm{g} / \mathrm{mL})$} \\
\hline $\mathrm{n}$ & 73 & 80 & 72 & 70 & 68 \\
\hline Baseline $^{a}$ & $4.83(3.48)$ & $5.99(3.75)$ & $6.79(4.53)$ & $6.59(4.36)$ & $6.23(4.92)$ \\
\hline Week $12^{\mathrm{a}}$ & $4.39(2.95)$ & $8.79(5.43)$ & $10.83(5.91)$ & $10.56(5.87)$ & $10.93(7.09)$ \\
\hline Change from baseline ${ }^{b}$ & $-0.25(-0.93,0.43)$ & $2.82(2.18,3.47)^{* *}$ & $3.96(3.28,4.64)^{* *}$ & $3.88(3.19,4.56)^{* *}$ & $4.67(3.97,5.36)^{* *}$ \\
\hline Change from placebo ${ }^{c}$ & -- & $3.07(2.14,4.00)^{\dagger \dagger}$ & $4.21(3.25,5.17)^{\dagger \dagger}$ & $4.12(3.15,5.09)^{\dagger \dagger}$ & $4.92(3.95,5.89)^{\dagger \dagger}$ \\
\hline \multicolumn{6}{|c|}{ Glycosylated Albumin (\%) } \\
\hline $\mathrm{n}$ & 73 & 80 & 72 & 70 & 68 \\
\hline Baseline $^{a}$ & $23.7(4.5)$ & $21.5(3.9)$ & $22.0(4.2)$ & $21.9(4.5)$ & $21.9(3.9)$ \\
\hline Week $12^{\mathrm{a}}$ & $24.2(4.9)$ & $19.7(3.7)$ & $19.4(4.1)$ & $19.3(4.4)$ & $19.0(3.0)$ \\
\hline Change from baseline ${ }^{\mathrm{b}}$ & $0.7(0.2,1.1)^{*}$ & $-1.9(-2.4,-1.5)^{* *}$ & $-2.6(-3.1,-2.2)^{* *}$ & $-2.6(-3.0,-2.1)^{* *}$ & $-2.9(-3.4,-2.5)^{* *}$ \\
\hline Change from placebo ${ }^{c}$ & -- & $-2.6(-3.2,-2.0)^{\dagger \dagger}$ & $-3.3(-3.9,-2.7)^{\dagger \dagger}$ & $-3.2(-3.9,-2.6)^{\dagger \dagger}$ & $-3.6(-4.3,-3.0)^{\dagger \dagger}$ \\
\hline \multicolumn{6}{|l|}{ HOMA- $\beta$ (\%) } \\
\hline $\mathrm{n}$ & 71 & 80 & 72 & 70 & 68 \\
\hline Baseline $^{\mathrm{a}}$ & $26.4(17.3)$ & $37.0(27.8)$ & $33.5(29.9)$ & $37.8(39.7)$ & $32.0(18.7)$ \\
\hline Week $12^{\mathrm{a}}$ & $28.0(19.2)$ & $45.6(34.4)$ & $43.5(31.9)$ & $52.5(54.8)$ & $42.6(23.5)$ \\
\hline Change from baseline ${ }^{\mathrm{b}}$ & $2.5(-1.4,6.4)$ & $8.3(4.7,11.9)^{* *}$ & $10.0(6.2,13.8)^{* *}$ & $14.1(10.2,18.0)^{* *}$ & $10.8(6.9,14.7)^{* *}$ \\
\hline Change from placebo $^{\mathrm{c}}$ & -- & $5.8(0.5,11.1)^{\dagger}$ & $7.5(2.1,13.0)^{\dagger}$ & $11.6(6.1,17.1)^{\dagger \dagger}$ & $8.3(2.8,13.8)^{\dagger}$ \\
\hline \multicolumn{6}{|c|}{ Postprandial Glycemic Endpoints } \\
\hline \multicolumn{6}{|c|}{ PP Glucose (120 min); mg/dL } \\
\hline $\mathrm{n}$ & 67 & 77 & 70 & 68 & 67 \\
\hline Baseline $^{\mathrm{a}}$ & $249.7(66.6)$ & $230.7(57.5)$ & $244.3(63.9)$ & $235.1(64.6)$ & 244.7 (71.9) \\
\hline Week $12^{\mathrm{a}}$ & $248.9(70.8)$ & $195.5(54.5)$ & $192.8(60.4)$ & $179.7(59.0)$ & $180.0(59.9)$ \\
\hline Change from baseline ${ }^{\mathrm{b}}$ & $1.7(-8.3,11.7)$ & $-38.6(-47.9,-29.3)^{* *}$ & $-50.5(-60.2,-40.7)^{* *}$ & $-56.6(-66.5,-46.7)^{* *}$ & $-63.4(-73.3,-53.4)^{* *}$ \\
\hline Change from placebo ${ }^{d}$ & -- & $-40.3(-53.9,-26.6)^{\dagger \dagger}$ & $-52.2(-66.1,-38.2)^{\dagger \dagger}$ & $-58.3(-72.4,-44.2)^{\dagger \dagger}$ & $-65.0(-79.1,-51.0)^{\dagger \dagger}$ \\
\hline PP Glucose $\mathrm{AUC}_{0-2 \mathrm{~h}} ; \mathrm{m}$ & & & & & \\
\hline $\mathrm{n}$ & 67 & 77 & 69 & 68 & 67 \\
\hline Baseline $^{a}$ & $472.1(90.9)$ & 440.7 (81.9) & $457.7(89.2)$ & $448.9(92.5)$ & $466.9(102.0)$ \\
\hline Week $12^{\mathrm{a}}$ & $477.0(99.0)$ & $393.3(76.9)$ & $381.4(91.5)$ & $367.5(84.0)$ & $366.3(76.8)$ \\
\hline Change from baseline ${ }^{b}$ & $9.6(-5.2,24.3)$ & $-53.5(-67.3,-39.7)^{* *}$ & $-76.2(-90.7,-61.7)^{* *}$ & $-83.2(-97.8,-68.5)^{* *}$ & $-96.9(-111.6,-82.2)^{* *}$ \\
\hline Change from placebo ${ }^{d}$ & -- & $-63.1(-83.3,-42.9)^{\dagger \dagger}$ & $-85.8(-106.4,-65.1)^{\dagger \dagger}$ & $-92.7(-113.6,-71.9)^{\dagger \dagger}$ & $-106.5(-127.2,-85.7)^{\dagger \dagger}$ \\
\hline PP Insulin $\mathrm{AUC}_{0-2 \mathrm{~h}} ; \mu \mathrm{U}$ & & & & & \\
\hline $\mathrm{n}$ & 67 & 77 & 70 & 68 & 67 \\
\hline Baseline $^{\mathrm{a}}$ & $55.8(27.1)$ & $81.7(55.0)$ & $72.0(49.9)$ & $82.5(62.6)$ & $76.5(45.8)$ \\
\hline Week $12^{\mathrm{a}}$ & $58.9(31.8)$ & $87.1(64.5)$ & $74.9(52.1)$ & $89.3(53.7)$ & $87.2(41.5)$ \\
\hline Change from baseline ${ }^{\mathrm{b}}$ & $0.1(-6.9,7.1)$ & $6.7(0.2,13.2)^{*}$ & $2.6(-4.2,9.4)$ & $8.2(1.3,15.1)^{*}$ & $11.1(4.1,18.0)^{*}$ \\
\hline Change from placebo $^{d}$ & -- & $6.6(-3.0,16.3)$ & $2.5(-7.2,12.3)$ & $8.1(-1.9,18.1)$ & $11.0(1.1,20.9)^{\dagger}$ \\
\hline PP C-peptide $\mathrm{AUC}_{0-2 \mathrm{~h}}$ & & & & & \\
\hline $\mathrm{n}$ & 67 & 77 & 70 & 68 & 67 \\
\hline Baseline $^{\mathrm{a}}$ & $8.49(2.49)$ & $10.02(3.27)$ & $9.43(3.25)$ & $9.68(3.04)$ & $9.72(2.62)$ \\
\hline Week $12^{\mathrm{a}}$ & $8.40(2.61)$ & $10.12(3.44)$ & $9.38(3.19)$ & $9.95(2.86)$ & $10.77(2.67)$ \\
\hline Change from baseline ${ }^{\mathrm{b}}$ & $-0.25(-0.66,0.16)$ & $0.19(-0.19,0.57)$ & $-0.05(-0.45,0.34)$ & $0.29(-0.11,0.70)$ & $1.09(0.68,1.49)^{* *}$ \\
\hline Change from placebo ${ }^{d}$ & -- & $0.44(-0.12,1.00)$ & $0.19(-0.38,0.76)$ & $0.54(-0.04,1.12)$ & $1.34(0.76,1.91)^{\dagger \dagger}$ \\
\hline Insulinogenic Index & & & & & \\
\hline $\mathrm{n}$ & 67 & 77 & 69 & 68 & 67 \\
\hline Baseline $^{\mathrm{a}}$ & $0.25(0.21)$ & $0.35(0.28)$ & $0.26(0.33)$ & $0.33(0.27)$ & $0.30(0.20)$ \\
\hline Week $12^{\mathrm{a}}$ & $0.25(0.17)$ & $0.46(0.45)$ & $0.44(0.46)$ & $0.65(1.42)$ & $0.56(0.39)$ \\
\hline Change from baseline ${ }^{b}$ & $-0.00(-0.16,0.16)$ & $0.12(-0.03,0.27)$ & $0.18(0.03,0.34)^{*}$ & $0.32(0.16,0.48)^{* *}$ & $0.26(0.10,0.42)^{*}$ \\
\hline Change from placebo ${ }^{d}$ & -- & $0.12(-0.10,0.34)$ & $0.19(-0.04,0.41)$ & $0.32(0.09,0.55)^{\dagger}$ & $0.26(0.04,0.49)^{\dagger}$ \\
\hline PP Glucagon $A \mathbf{U C}_{0-2 \mathrm{~h}}$ & & & & & \\
\hline $\mathrm{n}$ & 31 & 37 & 31 & 30 & 30 \\
\hline Baseline $^{\mathrm{a}}$ & $232.5(53.6)$ & $256.6(79.9)$ & $238.9(75.6)$ & $254.5(77.6)$ & $252.0(82.0)$ \\
\hline Week $12^{\mathrm{a}}$ & $235.1(53.5)$ & $236.7(71.6)$ & $213.1(76.9)$ & $226.1(78.8)$ & $241.8(89.2)$ \\
\hline Change from baseline ${ }^{\mathrm{b}}$ & $0.1(-14.0,14.1)$ & $-17.3(-30.1,-4.4)^{*}$ & $-27.4(-41.4,-13.4)^{* *}$ & $-23.7(-38.1,-9.2)^{*}$ & $-8.9(-23.2,5.3)$ \\
\hline Change from placebo ${ }^{d}$ & -- & $-17.3(-36.4,1.8)$ & $-27.5(-47.3,-7.6)^{\dagger}$ & $-23.7(-44.0,-3.5)^{\dagger}$ & $-9.0(-29.0,11.1)$ \\
\hline
\end{tabular}


(a)

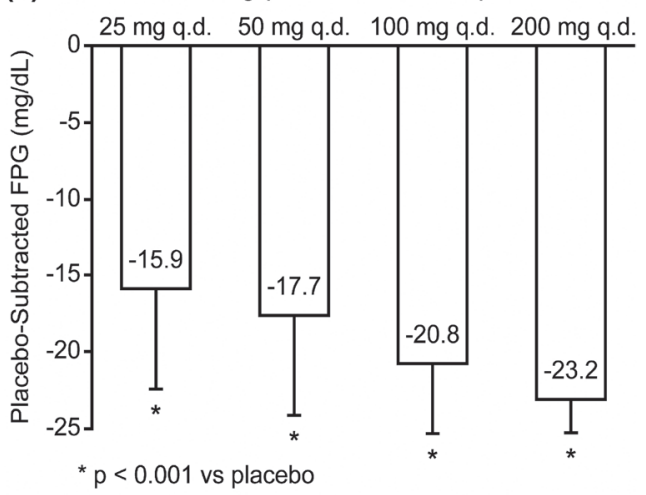

(b) Sitagliptin Treatment Group

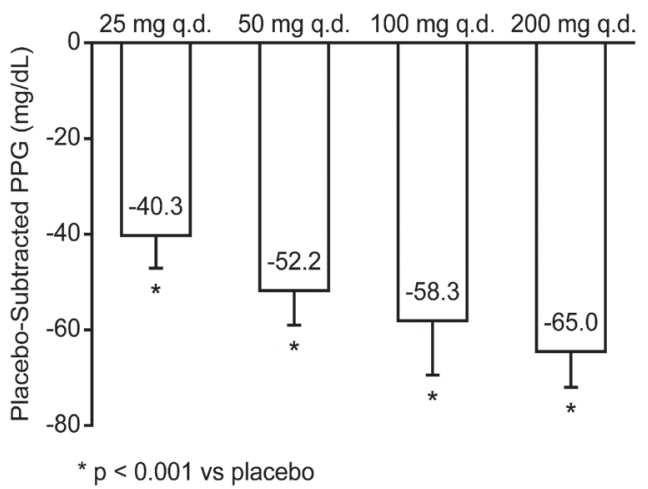

Fig. 3. Effect of sitagliptin on (a) fasting plasma glucose (PPG) and (b) 2-hr postprandial glucose (PPG) following 12 weeks of treatment. Results are presented as placebosubtracted change from baseline.

tively) and with $50 \mathrm{mg}$ compared with $200 \mathrm{mg}$ $(p=0.049)$ (data not shown). Of all the sitagliptin doses tested, only the 200-mg group produced significant increases from baseline in insulin $\mathrm{AUC}_{0-2 \mathrm{hr}}$ $(p=0.030)$ and C-peptide $\mathrm{AUC}_{0-2 \mathrm{hr}}(p<0.001)$ compared with placebo. The insulinogenic index was significantly increased with sitagliptin 100 and 200 mg compared with placebo $(p<0.050)$. The effects of sitagliptin on plasma glucagon levels were examined in a subset of patients enrolled at sites equipped to appropriately collect and process the samples. Although the predefined stepwise linear contrast test that included the $200-\mathrm{mg}$ dose was not significant, treatment with sitagliptin 25,50 and $100 \mathrm{mg}$ led to significant $(p<0.001$ in $50 \mathrm{mg}, p<0.05$ in 25 and 100 $\mathrm{mg}$ ) reductions from baseline in glucagon $\mathrm{AUC}_{0-2} \mathrm{hr}$ Significant reductions from baseline relative to placebo were observed for the 50- and 100-mg doses of sitagliptin $(p<0.05)$.

\section{Safety}

Compared with the placebo group, the incidences of overall clinical AEs were similar in the 25-mg group, with a dose-related trend toward an increased incidence across the 50-, 100-, and 200-mg groups (Table 3). There were similar proportions of patients across the placebo, sitagliptin 25-, 50-, 100-, and 200$\mathrm{mg}$ groups for whom infections and infestations disorder AEs were reported (20 [27.4\%], 17 [21.3\%], 22 [30.6\%], 29 [41.4\%], 18 [26.5\%], respectively); there was no dose-dependent increase in the proportion of patients in these groups for whom gastrointestinal disorders AEs were reported (9 [12.3\%], 13 [16.3\%], 13 [18.1\%], 9 [12.9\%], 9 [13.2\%], respectively), with similar incidences observed for the placebo, 100-, and 200-mg groups. The most commonly reported clinical AE was nasopharyngitis, which had a similar incidence across the placebo and sitagliptin groups (17 [23.3\%], 16 [20.0\%], 16 [22.2\%], 23 [32.9\%] and 12 [17.6\%] for the placebo, sitagliptin 25-, 50-, 100-, and 200-mg groups, respectively). The incidences of hypoglycemia and selected gastrointestinal AEs across the sitagliptin groups were low and comparable to that seen in the placebo group (Table 3). Only one patient in this study (a patient in the 200-mg group) discontinued due to a clinical AE; a clinical AE of myocardial ischemia determined by the investigator to be "definitely not related" to study medication.

The incidences of drug-related clinical AEs were generally similar between the placebo and the individual sitagliptin groups, with no apparent dose-related trend (Table 3 ). There were no clinically meaningful differences in the incidences of specific drug-related clinical AEs across the groups (Table 3). No patients in this study discontinued due to drug-related clinical AEs (Table 3). There were 3 patients for whom 5 serious AEs were reported.

All serious AEs were determined by the investigator to be "definitely not related" to study medication: 1 patient in the 50-mg group (angina pectoris) and 2 patients in the 200-mg group (one patient had an overdose and 1 patient reported AEs of cardiac failure, hypertensive heart disease, and myocardial ischemia). Per protocol, overdose was defined as a serious AE, regardless of whether an adverse event was associated with the overdose. The patient for whom an AE of overdose was reported took sitagliptin $400 \mathrm{mg} /$ day for one day without any associated adverse signs or symptoms. No patients discontinued due to serious clinical AEs. 
Table 3. Summary of adverse events

\begin{tabular}{|c|c|c|c|c|c|}
\hline \multirow[b]{3}{*}{ Adverse events } & \multicolumn{5}{|c|}{ Number (\%) of patients } \\
\hline & \multirow[b]{2}{*}{$\begin{array}{c}\text { Placebo } \\
n=73\end{array}$} & \multicolumn{4}{|c|}{ Sitagliptin } \\
\hline & & $\begin{array}{c}25 \mathrm{mg} \text { q.d. } \\
\mathrm{n}=80\end{array}$ & $\begin{array}{c}50 \mathrm{mg} \text { q.d. } \\
\mathrm{n}=72\end{array}$ & $\begin{array}{c}100 \text { mg q.d. } \\
n=70\end{array}$ & $\begin{array}{c}200 \mathrm{mg} \text { q.d } \\
\mathrm{n}=68\end{array}$ \\
\hline One or more clinical AEs & $39(53.4)$ & $46(57.5)$ & $47(65.3)$ & $46(65.7)$ & $50(73.5)$ \\
\hline Serious clinical AEs & $0(0.0)$ & $0(0.0)$ & $1(1.4)$ & $0(0.0)$ & $2(2.9)$ \\
\hline Drug-related clinical SAEs ${ }^{\mathrm{a}}$ & $0(0.0)$ & $0(0.0)$ & $0(0.0)$ & $0(0.0)$ & $0(0.0)$ \\
\hline Discontinued due to clinical AEs & $0(0.0)$ & $0(0.0)$ & $0(0.0)$ & $0(0.0)$ & $1(1.5)$ \\
\hline Discontinued due to drug-related clinical AEs & $0(0.0)$ & $0(0.0)$ & $0(0.0)$ & $0(0.0)$ & $0(0.0)$ \\
\hline Discontinued due to clinical SAEs & $0(0.0)$ & $0(0.0)$ & $0(0.0)$ & $0(0.0)$ & $0(0.0)$ \\
\hline \multicolumn{6}{|l|}{ Clinical AEs of interest } \\
\hline Hypoglycemia & $2(2.7)$ & $1(1.3)$ & $3(4.2)$ & $3(4.3)$ & $3(4.4)$ \\
\hline Gastrointestinal $\mathrm{AEs}^{\mathrm{b}}$ & $1(1.4)$ & $3(3.8)$ & $0(0.0)$ & $0(0.0)$ & $3(4.4)$ \\
\hline Clinical drug related adverse events ${ }^{a}$ & $3(4.1)$ & $6(7.5)$ & $3(4.2)$ & $5(7.1)$ & $1(1.5)$ \\
\hline Anemia & $0(0.0)$ & $0(0.0)$ & $1(1.4)$ & $0(0.0)$ & $0(0.0)$ \\
\hline Bundle branch block right ${ }^{\mathrm{c}}$ & $0(0.0)$ & $1(1.3)$ & $0(0.0)$ & $0(0.0)$ & $0(0.0)$ \\
\hline Palpitations $^{\mathrm{c}}$ & $0(0.0)$ & $1(1.3)$ & $0(0.0)$ & $0(0.0)$ & $0(0.0)$ \\
\hline Constipation & $0(0.0)$ & $2(2.5)$ & $0(0.0)$ & $0(0.0)$ & $0(0.0)$ \\
\hline Rectal polyp & $0(0.0)$ & $0(0.0)$ & $1(1.4)$ & $0(0.0)$ & $0(0.0)$ \\
\hline Stomatitis & $0(0.0)$ & $1(1.3)$ & $0(0.0)$ & $0(0.0)$ & $0(0.0)$ \\
\hline ECG T wave amplitude decreased & $0(0.0)$ & $1(1.3)$ & $0(0.0)$ & $0(0.0)$ & $0(0.0)$ \\
\hline ECG T wave inversion & $1(1.4)$ & $0(0.0)$ & $0(0.0)$ & $0(0.0)$ & $0(0.0)$ \\
\hline Weight decreased & $1(1.4)$ & $0(0.0)$ & $0(0.0)$ & $0(0.0)$ & $0(0.0)$ \\
\hline Hypoglycemia & $1(1.4)$ & $0(0.0)$ & $1(1.4)$ & $3(4.3)$ & $1(1.5)$ \\
\hline Dizziness postural & $0(0.0)$ & $0(0.0)$ & $0(0.0)$ & $1(1.4)$ & $0(0.0)$ \\
\hline Neuropathy peripheral & $0(0.0)$ & $0(0.0)$ & $0(0.0)$ & $1(1.4)$ & $0(0.0)$ \\
\hline Epistaxis & $0(0.0)$ & $1(1.3)$ & $0(0.0)$ & $0(0.0)$ & $0(0.0)$ \\
\hline One or more laboratory AEs & $8(11.0)$ & $15(18.8)$ & $14(19.4)$ & $12(17.1)$ & $8(11.8)$ \\
\hline Drug-related laboratory AEs & $2(2.7)$ & $3(3.8)$ & $6(8.3)$ & $1(1.4)$ & $2(2.9)$ \\
\hline Serious laboratory AEs & $0(0.0)$ & $0(0.0)$ & $0(0.0)$ & $0(0.0)$ & $0(0.0)$ \\
\hline Discontinued due to laboratory AEs & $0(0.0)$ & $0(0.0)$ & $1(1.4)$ & $1(1.4)$ & $0(0.0)$ \\
\hline Discontinued due to drug-related laboratory AEs & $0(0.0)$ & $0(0.0)$ & $1(1.4)$ & $0(0.0)$ & $0(0.0)$ \\
\hline
\end{tabular}

${ }^{\mathrm{a} D e t e r m i n e d ~ b y ~ t h e ~ i n v e s t i g a t o r ~ t o ~ b e ~ p o s s i b l y, ~ p r o b a b l y, ~ o r ~ d e f i n i t e l y ~ d r u g-r e l a t e d ; ~}{ }^{\mathrm{b}}$ Redefined category includes gastrointestinal AEs of nausea, vomiting, and diarrhea; 'One patient in the 25-mg group experienced two drug-related clinical adverse experiences of "bundle branch block right" and "palpitations" during the 12-week study. This patient was counted once in the overall tally of total drug-related clinical adverse experiences; $\mathrm{AE}=$ adverse event; $\mathrm{ECG}=$ electrocardiogram; q.d. = once daily; $\mathrm{SAE}=$ serious adverse event

There was no dose-dependent increase in the proportion of patients in these groups for whom laboratory AEs were reported (8 (11.0\%), 15 (18.8\%), $14(19.4 \%), 12(17.1 \%)$, and $8(11.8 \%)$ in the placebo, sitagliptin 25-, 50-, 100-, and 200-mg groups, respectively (Table 3), with similar incidences observed for the placebo and 200-mg groups. Among the specific laboratory AEs, a total of 4 patients in the 50-mg group experienced elevations in ALT (4 [5.6\%]) and AST (3 [4.2\%]) versus none in the higher-dose groups. There was a higher incidence of creatine kinase elevations in the $25 \mathrm{-mg}$ group (7 [8.8\%]) compared with the other treatment groups (2 [2.7\%], 2 [2.8\%], 1 [1.4\%], and 1 [1.5\%] for placebo, sitaglip- tin $50 \mathrm{mg}, 100 \mathrm{mg}$, and $200 \mathrm{mg}$, respectively). Two $(2.5 \%), 3(4.3 \%)$, and $3(4.4 \%)$ of patients in the sitagliptin 25-, 100-, and 200-mg groups were reported to have an $\mathrm{AE}$ of proteinuria compared with none in the placebo and sitagliptin 50-mg groups. There was no evidence of a dose-related trend toward increased incidences of laboratory AEs across the sitagliptin groups. The incidences of drug-related laboratory AEs were low and generally similar across the placebo, sitagliptin 25-, 100-, and 200-mg groups (2 [2.7\%], 3 [3.8\%], $1[1.4 \%]$ and 2 [2.9\%], respectively), with a slightly higher rate in the $50 \mathrm{mg}$ group (6 [8.3\%]). All laboratory AEs resolved spontaneously without any medical intervention; 2 patients discontinued study medication 
prematurely due to laboratory AEs.

At Week 12, treatment with sitagliptin had no clinically meaningful effect on body weight relative to baseline or placebo. The placebo group showed a small non-significant reduction from baseline in body weight of $-0.5 \mathrm{~kg}(95 \% \mathrm{CI}:-0.8,-0.1 ; p=0.005)$, whereas slight increases of $0.4 \mathrm{~kg}(95 \% \mathrm{CI}: 0.1,0.7$; $p<0.050), 0.3 \mathrm{~kg}(95 \%$ CI: $0.0,0.6 ; p<0.050), 0.1 \mathrm{~kg}$ (95\% CI: $-0.2,0.5 ; p=0.386)$, and $0.3 \mathrm{~kg}(95 \% \mathrm{CI}$ : $0.0,0.6 ; p=0.079)$ were observed across the sitagliptin 25-, 50-, 100-, and 200-mg groups, respectively. The changes in body weight observed with sitagliptin treatment did not appear to be dose related. No clinically meaningful mean changes from baseline in vital signs were observed in any treatment group.

\section{Discussion}

This 12-week, placebo-controlled, randomized study evaluated the glycemic efficacy, dose-response and safety/tolerability profile of sitagliptin doses ranging from 25 to $200 \mathrm{mg}$ q.d. in Japanese patients with T2DM who had inadequate glycemic control with diet and exercise therapy alone. Treatment with sitagliptin monotherapy led to significant dose-related and clinically meaningful reductions in $\mathrm{HbA}_{1 \mathrm{c}}$ relative to placebo after 12 weeks of treatment. The 50-mg dose of sitagliptin was maximally effective at reducing $\mathrm{HbA}_{1 c}$, with similar magnitude reductions observed in the $100-\mathrm{mg}$ group and only small numerically greater reductions in the 200-mg group. This finding suggests that, in Japanese patients with T2DM, starting treatment with the 100- and 200-mg doses of sitagliptin does not provide further, clinically meaningful improvements in $\mathrm{HbA}_{1 \mathrm{c}}$ beyond that seen with the 50$\mathrm{mg}$ dose in this treatment period. The improvements in $\mathrm{HbA}_{1 \mathrm{c}}$ observed in this study of Japanese patients with T2DM were maintained over the 12-week treatment period for all sitagliptin doses, whereas deterioration was observed in the placebo group. A previous, longer-term study conducted in non-Japanese patients with T2DM demonstrated that sitagliptin doses of 100 and $200 \mathrm{mg}$ produced maximum reductions in $\mathrm{HbA}_{\mathrm{lc}}$ by Week 12 with no further improvements observed out to week 18 [14].

In addition to reductions in $\mathrm{HbA}_{1 \mathrm{c}}$, treatment with sitagliptin also provided significant dose-dependent improvements in other important glycemic endpoints compared with placebo, including FPG, 2-hr
PPG, glucose $\mathrm{AUC}_{0-2 \mathrm{hr}}, 1,5-\mathrm{AG}$, and GA. For FPG, 2-hr PPG, and glucose $\mathrm{AUC}_{0-2 \mathrm{hr}}$; stepwise numerically greater improvements were observed across the entire dose range, suggesting that some patients in this population may receive additional benefit from a higher dose of sitagliptin. The 50 -mg dose produced significantly greater improvements in 1,5-AG and GA relative to the $25-\mathrm{mg}$ dose, with no further improvements observed at doses of 100 and $200 \mathrm{mg}$. The combined improvements in FPG, 2-hr PPG and glucose $\mathrm{AUC}_{0-2 \mathrm{hr}}$ observed in this study of Japanese patients demonstrates that sitagliptin provides clinically meaningful glucose-lowering efficacy in both the fasting and postprandial states.

All sitagliptin doses provided numerical increases relative to placebo in insulin $\mathrm{AUC}_{0-2 \mathrm{hr}}$, although statistical significance was reached at the 200-mg dose only. The effects of sitagliptin therapy on plasma insulin levels in the context of lowered glucose excursion suggest that sitagliptin may enhance the insulin response following a meal. Significant improvements in $\beta$-cell function were observed with all sitagliptin doses relative to placebo using both fasting and postprandial measurements (i.e., HOMA- $\beta$ and insulinogenic index). This study also assessed the effects of sitagliptin on plasma glucagon levels in a subset of patients enrolled at clinic sites equipped to adequately collect and process the samples for this assay. Treatment with sitagliptin 25,50 , or $100 \mathrm{mg}$ led to significant reductions from baseline in glucagon $\mathrm{AUC}_{0-2 \mathrm{hr}}$, although there was no evidence of a doserelated trend in the glucagon response. Significant reductions from baseline in glucagon $\mathrm{AUC}_{0-2 \mathrm{hr}}$ were also observed with sitagliptin 50 and $100 \mathrm{mg}$ relative to placebo. Consistent with the glucagon-lowering previously reported by Herman et al. [5], this finding suggests that sitagliptin alters the secretion of glucagon by the $\alpha$-cell, although further research may better elucidate the effects of sitagliptin on hepatic glucose output. Consistent with prior findings in non-Japanese patients, there was no significant effect of sitagliptin treatment compared with placebo on HOMA-IR in the current study (data not shown), suggesting that sitagliptin does not affect peripheral insulin sensitivity [10, $11,13,14]$.

In this study, there were numerical incremental improvements in both FPG and 2-hr PPG with sitagliptin doses of $100(\mathrm{~N}=70)$ and $200 \mathrm{mg}(\mathrm{N}=68)$ relative to the 50-mg dose $(\mathrm{N}=72)$; however, the differences ob- 
served between these treatment groups were not statistically significant. Collectively, these observations suggest that the 50-mg dose provided near maximal fasting and postprandial glycemic efficacy in Japanese patients, thus supporting sitagliptin $50 \mathrm{mg}$ as the primary dose in the Phase III development program in Japan. Since numerically larger reductions in both FPG and 2-hr PPG were seen in this study with increasing doses of sitagliptin, further investigation is warranted to determine the possible increased glycemic efficacy of sitagliptin doses greater than $50 \mathrm{mg}$ in Japanese patients with T2DM. The results of the present study in Japanese patients with T2DM are consistent with those observed in previous monotherapy studies conducted in non-Japanese patients with T2DM that demonstrated some improvement in glycemic parameters with $100 \mathrm{mg}$ compared with $50 \mathrm{mg}$ of sitagliptin [10,11].

Sitagliptin was generally well-tolerated in this study of Japanese patients with T2DM. Although the overall AE rate was somewhat higher in the sitagliptin treatment groups compared with placebo, drugrelated AEs and discontinuations due to drug-related AEs occurred at a low and generally similar rate across treatment groups. A very low incidence of hypoglycemia was observed with sitagliptin treatment despite the effective fasting and postprandial glucose control seen in this study. The incidence of hypoglycemia observed in the sitagliptin groups did not appear to be meaningfully different from that seen in the placebo group. This finding is consistent with evidence indicating that GLP-1 stimulates insulin release in a glucose-dependent manner [18]. The incidence of gastrointestinal AEs was evaluated in this study since sitagliptin increases the level of active GLP-1 in the plasma and GLP-1 analogues are known to be associated with symptoms of nausea, vomiting, and diarrhea [19]. In this study, the incidence of gastrointestinal AEs was very low with no clinically meaningful differences between the sitagliptin and placebo groups. Finally, treatment with sitagliptin had a generally neutral effect on body weight without evidence of a doserelated increase. In conclusion, this 12 -week study in Japanese patients with T2DM and inadequate glycemic control on diet and exercise therapy alone demonstrated that once-daily sitagliptin at doses of 25, 50, 100 , and $200 \mathrm{mg}$ provided statistically significant and clinically important reductions in $\mathrm{HbA}_{1 \mathrm{c}}, \mathrm{FPG}$, and 2-hr PPG as well as significant improvements in indices of insulin secretion and $\beta$-cell function, including HOMA- $\beta$ and insulinogenic index. Treatment with sitagliptin was well tolerated and was associated with a low rate of hypoglycemia as well as a neutral effect on body weight.

\section{Acknowledgments}

The authors gratefully acknowledge Amy O. Johnson Levonas for her assistance with preparation of this manuscript.

\section{References}

1. Ahren B (2003) Gut peptides and type 2 diabetes mellitus treatment. Curr Diab Rep 3: 365-372.

2. Drucker DJ (2002) Biological actions and therapeutic potential of the glucagon-like peptides. Gastroenterology 122: 531-544.

3. Mentlein R, Gallwitz B, Schmidt WE (1993) Dipeptidyl-peptidase IV hydrolyses gastric inhibitory polypeptide, glucagon-like peptide-1(7-36)amide, peptide histidine methionine and is responsible for their degradation in human serum. Eur J Biochem 214: 829835 .

4. Deacon CF, Johnsen AH, Holst JJ (1995) Degradation of glucagon-like peptide-1 by human plasma in vitro yields an N-terminally truncated peptide that is a major endogenous metabolite in vivo. J Clin Endocrinol Metab 80: 952-957.
5. Herman GA, Stein PP, Thornberry NA, Wagner JA (2007) Dipeptidyl peptidase-4 inhibitors for the treatment of type 2 diabetes: focus on sitagliptin. Clin Pharmacol Ther 81: 761-767.

6. Herman GA, Stevens C, Van Dyck K, Bergman A, Yi B, De Smet M, Snyder K, Hilliard D, Tanen M, Tanaka W, Wang AQ, Zeng W, Musson D, Winchell G, Davies MJ, Ramael S, Gottesdiener KM, Wagner JA (2005) Pharmacokinetics and pharmacodynamics of sitagliptin, an inhibitor of dipeptidyl peptidase IV, in healthy subjects: results from two randomized, double-blind, placebo-controlled studies with single oral doses. Clin Pharmacol Ther 78: 675-688.

7. Bergman AJ, Stevens C, Zhou Y, Yi B, Laethem M, De Smet M, Snyder K, Hilliard D, Tanaka W, Zeng W, Tanen M, Wang AQ, Chen Li, Winchell G, Davies 
MJ, Ramael S, Wagner JA, Herman GA (2006) Pharmacokinetic and pharmacodynamic properties of multiple oral doses of sitagliptin, a dipeptidyl peptidase-IV inhibitor: a double-blind, randomized, placebo-controlled study in healthy male volunteers. Clin Ther 28: 55-72.

8. Herman GA, Bergman A, Stevens C, Kotey P, Yi B, Zhao P, Dietrich B, Golor G, Schrodter A, Keymeulen B, Lasseter KC, Kipnes MS, Snyder K, Hilliard D, Tanen M, Cilissen C, De Smet M, de Lepeleire I, Van Dyck K, Wang AQ, Zeng W, Davies MJ, Tanaka W, Holst JJ, Deacon CF, Keith M. Gottesdiener KM, Wagner JA (2006) Effect of single oral doses of sitagliptin, a dipeptidyl peptidase-4 inhibitor, on incretin and plasma glucose levels after an oral glucose tolerance test in patients with type 2 diabetes. J Clin Endocrinol Metab 91: 4612-4619.

9. Herman GA, Bergman A, Liu F, Stevens C, Wang AQ, Zeng W, Chen L, Snyder K, Hilliard D, Tanen M, Tanaka W, Meehan AG, Lasseter K, Dilzer S, Blum R, Wagner JA (2006) Pharmacokinetics and pharmacodynamic effects of the oral DPP-4 inhibitor sitagliptin in middle-aged obese subjects. J Clin Pharmacol 46: 876-886.

10. Scott R, Wu M, Sanchez M, Stein P (2007) Efficacy and tolerability of the dipeptidyl peptidase-4 inhibitor sitagliptin as monotherapy over 12 weeks in patients with type 2 diabetes. Int J Clin Pract 61: 171-180.

11. Hanefeld M, Herman GA, Wu M, Mickel C, Sanchez M (2007) Once-daily sitagliptin, a dipeptidyl peptidase-4 inhibitor, for the treatment of patients with type 2 diabetes. Curr Med Res Opin 23: 1329-1339.

12. Product information. Januvia (sitagliptin) tablets. Whitehouse Station: Merck \& Co., Inc. 2007.
13. Aschner P, Kipnes MS, Lunceford JK, Sanchez M, Mickel C, Williams-Herman DE (2006) Effect of the dipeptidyl peptidase-4 inhibitor sitagliptin as monotherapy on glycemic control in patients with type 2 diabetes. Diabetes Care 29: 2632-2637.

14. Raz I, Hanefeld M, Xu L, Caria C, Williams-Herman D, Khatami H (2006) Efficacy and safety of the dipeptidyl peptidase-4 inhibitor sitagliptin as monotherapy in patients with type 2 diabetes mellitus. Diabetologia 49: 2564-2571.

15. Bergman AJ, Cote J, Yi B, Marbury T, Swan SK, Smith W, Gottesdiener K, Wagner JA, Herman GA (2007) Effect of renal insufficiency on the pharmacokinetics of sitagliptin, a dipeptidyl peptidase-4 inhibitor. Diabetes Care 30: 1862-1864.

16. Chan JC, Scott R, Arjona Ferreira JC, Sheng D, Gonzalez E, Davies MJ, Stein PP, Kaufman KD, Amatruda JM, Williams-Herman D (2008) Safety and efficacy of sitagliptin in patients with type 2 diabetes and chronic renal insufficiency. Diabetes Obes Metab 10: 545-555.

17. Nonaka K, Kakikawa T, Sato A, Okuyama K, Fujimoto G, Kato N, Hideyo Suzuki H, Hirayama Y, Ahmed T, Davies MJ, Stein PP (2008) Efficacy and safety of sitagliptin monotherapy in Japanese patients with type 2 diabetes. Diabetes Res Clin Pract 79: 291-298.

18. Nauck MA, Kleine N, Orskov C, Holst JJ, Willms B, Creutzfeldt W (1993) Normalization of fasting hyperglycaemia by exogenous glucagon-like peptide 1 (7-36 amide) in type 2 (non-insulin-dependent) diabetic patients. Diabetologia 36: 741-744.

19. Mikhail N (2006) Exenatide: a novel approach for treatment of type 2 diabetes. South Med J 99: 1271-1279.

\section{Appendix}

The Sitagliptin Study 044 Investigators

Sumio Kuniyoshi (Oroku Hospital), Isao Shiroma (Chibana Clinic), Kazuo Satake (Fukui General Hospital), Shoichi Akazawa (ShinKoga Hospital), Yoshiharu Kitakaze (Takamori Clinic), Taihei Murakami (Murakami Gastrointestinal Clinic), Mikio Suzuki (Sendai East Neurosurgical Hospital), Keiichi Oikawa (Oikawa Medical Clinic), Hiroki Kamata (Kamata Medical Clinic), Yukihiro Nagai (Nagai Internal Medicine Clinic), Muneatsu Toshima (Niitsu Medical Center Hospital), Makoto Hayashi (Matsunami General Hospital), Yasuo Tanno (Tozai Clinic Sendai), Yasuhiko Ieki (Kurobe City Hospital), Masakazu Mizutani (Kozawa Eye Hospital), Shinya Nakamoto (Nakamoto Medical Clinic), Hiroshi Ohashi (Oyama East Clinic), Akira Kanamori (Kanamori Diabetes Clinic), Fumihiko Hojyo (Kobari General Clinic), Akira Yamauchi (Suruga Clinic), Toshihiro Kojima (Gifu Central Hospital), Satoshi Sasaki (Sasaki Medical Clinic), Toshiki Fujita (Biwako Ohashi Hospital), Tsuguyoshi Asano (Asano Kanamachi Clinic),
Hideki Hayashi (Koga Hospital 21), Tetsuaki Inokuchi (Inokuchi Clinic), Kazuhiro Harada (Kasaoka Daiichi Hospital), Woohyeon Lee (Asahi Clinic), Haruko Kunii (Kunii Hospital), Miyuki Sugimoto (Yamauchi Hospital), Akira Sanada (Sanada Clinic), Hiroshi Yamamoto (Yamamoto Medical Clinic), Yoshiki Iida (Iida Medical Clinic), Tetsuya Moriai (Megumino Hospital), Masatomo Sekiguchi (Sapporo-Kosei General Hospital), Shuichi Maeda (Kurosawa Hospital), Tetsuya Noto (Hakodate Kyouritsu Medical Hospital), Yuji Nakatani (Nakatani Hospital), Sho Yunoki (Miwa Memorial Hospital), Minoru Muta (Muta Medical Clinic), Atsumi Osaka (Kyomachi Medical Hospital), Noriko Omura (Nishinomiya Kyoritsu Neurosurgical Hospital), Yoichi Ehara (Yoshii Central Clinic), Mikio Matsumoto (Matsumoto Clinic), Toshiya Sato (Sato Medical Hospital), Hideo Manaka (Sagae City Hospital), Kazuko Saito (Seiryo Medical Clinic), Shuro Kondo (Tokyo Clinical Research Organisation for Medicine Clinic), Sachihiko Ozawa (Shimo-Okutama Clinic), Hiroyuki Fukita (Midorinofukita 
Clinic), Masataka Karube (New Medical Research System Clinic), Shunroku Sugimoto (Sugimoto Hospital), Takashi Saga (Tanaka Hospital), Yasuhiko Iwamoto (Tokyo Women's Medical University Hospital), Yasuo Nishimura (Veritas Hospital), Manabu Oyama (Shinsuma General Hospital), Munetoshi Matoba (Matoba Hospital), Yoshifumi Kanemaru (Kanemaru Medical Clinic), Mikio Fujioka (Fujioka Clinic), Shintaro Shokoku (Okayama Daiichi Hospital), Hiroaki Furuya (Koganei Chuo Hospital), Kazuya Yoshii (Izumino Hospital), Shigeru Fujii (Fukuoka Shin Mizumaki Hospital), Akio Urata (Urata Clinic), Manabu Shizume (Niizashiki Chuo General Hospital), Nobuhiro Yamada (Tsukuba University Hospital), Etsuo Otomo (Otomo Medical Clinic), Harumi Nishimura (Haru Clinic), Keizo Omori (Fukuoka Wajiro Hospital), Toyoshi Inoguchi (Kyusyu University Hospital), Takehiro Miyagishi (Miyagishi Hospital), Osamu Iwata (Shiodomeoak Clinic), Akihiko Tanaka (Toda Chuo General Hospital), Akira Shimada (Keio University Hospital), Hirokazu Shoda (Shoda Hospital), Daisuke Chujo (Kanazawa Social Insurance Hospital),
Tomohiro Ito (Obihiro-Kosei General Hospital), Shinichiro Ohashi (Clinic Komatsu), Nobuo Inokuchi (Inokuchi Medical Clinic), Kuninobu Nakatsuru (Miyazaki Zenjinkai Hospital), Yumi Miyazono (Shiminnomori Hospital), Jun Watanabe (Yukuhashi Central Hospital), Tsutomu Hirano (Showa University Higashi Hospital), Makoto Kajiyama (Uno Hospital), Shiro Nakayama (Nakayama Internal Medicine Rheumatism Allergy Clinic), Daigaku Uchida (Kimitsu Chuo Hospital), Tetsuro Sekiguchi (Sekiguchi Clinic), Motonobu Anai (Marunouchi Hospital attached to the Institute for Adult Diseases, Asahi Life Foundation), Shigehiro Katayama (Saitama Medical University Hospital), Kazuyuki Tobe (The University of Tokyo Hospital), Shoichiro Nagasaka (Jichi Medical University Hospital), Hisamitsu Ishihara (Tohoku University Hospital), Kazuya Yamagata (Osaka University Hospital), Masaki Shinomiya (Nishifuna Naika), Kazuo Yagi (Chiba University Hospital), Kazunori Yamada (Kyoto Medical Center), and Takeshi Nishikawa (Kumamoto University Hospital). 